\title{
Time course of platelet $a$ granule release in acute myocardial infarction treated with streptokinase
}

\author{
Niels Jørgen Frandsen, Kaj Winther, Flemming Pedersen, Ib Christiansen, Peter McNair
}

\begin{abstract}
Objective-To determine the time course of platelet $a$ granule release in patients with acute myocardial infarction treated with streptokinase.

Design-A prospective study.

Setting-Coronary care unit.

Patients-Nine with myocardial infarction treated with both streptokinase and aspirin, and nine with acute chest pain but without myocardial infarction, who were treated with aspirin only.

Methods-All patients received $250 \mathrm{mg}$ aspirin on admission and $150 \mathrm{mg}$ once daily thereafter. All patients who fulfilled the indications for streptokinase received 1.5 megaunits, in a single infusion. After the initial medication, serial measurements of plasma $\beta$ thromboglobulin and plasma platelet factor 4 were performed at fixed intervals after the onset of chest pain. The primary endpoint sought was the peak value of $\beta$ thromboglobulin and platelet factor 4 in each individual.
\end{abstract}

Results-The median peak plasma $\beta$ thromboglobulin in the infarction group was substantially higher than in those without infarction, at 37 (range 12 to 210) $v$ 15 (9 to 36$) \mathrm{mg} /$ litre, $P<0.01$. The corresponding values for plasma platelet factor 4 were $4.6(2.4$ to 60.0$) v 2.2(<2$ to 8.5$)$ mg/litre, $P<0.01$. Increased values were seen only within the first $12 \mathrm{~h}$ after onset of chest pain, and after $12 \mathrm{~h}$ there was no difference between the patients with myocardial infarction and those without. Aspirin treatment did not abolish $a$ granule release.

Conclusions-In patients with acute myocardial infarction treated with streptokinase the content of the $a$ granules is released within the first $12 \mathrm{~h}$ after the onset of chest pain. Aspirin apparently does not abolish this release.

(Heart 1996;75:141-144)

Keywords: platelet a granule; myocardial infarction; streptokinase; aspirin

There is some indication that streptokinase, used in myocardial infarction primarily as a fibrinolytic agent, can also induce release from platelet granules. ${ }^{12}$ However, the published evidence on this, and on the mooted capacity of myocardial infarction alone to induce granule release, ${ }^{3-6}$ is conflicting, and muddied by the uncertainty of what constitutes a reliable index of platelet release and by dependence on "before and after" samples taken at unstandardised times.

In view of the important practical implications of platelet release, and in particular its possible involvement in the development of reocclusion after thrombolytic treatment ${ }^{7-9}$ we have undertaken a detailed study of the time course of the process in patients with acute myocardial infarction treated with a single infusion of streptokinase. The opportunity was taken to make the study more definitive by applying the stringent measurement criteria of Kaplan and Owen, ${ }^{111}$ who proposed that a reliable index of granule release can only be obtained by measuring the plasma levels of both $\beta$ thromboglobulin and platelet factor 4 one alone will not do.

\section{Methods}

STUDY PROTOCOL

The study was approved by the local ethics committee. All patients admitted with suspected myocardial infarction from the first of October 1992 and 10 weeks ahead were included consecutively. Inclusion criteria were: age over 18 years, chest pain for less than $12 \mathrm{~h}$, and informed consent. Patients with serum creatine $>130 \mathrm{mmol} /$ litre were excluded. All patients received $250 \mathrm{mg}$ aspirin on admission, and $150 \mathrm{mg}$ once daily thereafter. If streptokinase was indicated, the patient received 1.5 megaunits over $1 \mathrm{~h}$. The diagnosis "myocardial infarction" was based on an increase in the $\mathrm{MB}$ fraction of creatine kinase above 10 units/litre. Ten healthy nonsmokers not using aspirin were used as controls.

\section{BLOOD SAMPLING AND LABORATORY} INVESTIGATIONS

Blood was always sampled after the treatment had been instituted. Samples were taken 3, 6, 9 , and $12 \mathrm{~h}$ and 1,3 , and $7 \mathrm{~d}$ after the onset of chest pain. Because of practical limitations, none of the patients was measured on all seven occasions. The mean number of blood tests per patient was three (range two to five).

Blood samples were drawn at the bedside with a 21 gauge needle, using minimum stasis, directly into Vacutainer tubes, which contained theophylline, adenosine, and prosta- 
Age, sex, serum total creatine kinase, and serum creatine kinase $M B$ fraction of the study groups

\begin{tabular}{lccll}
\hline & $M I$ & Non-MI & Controls & $P$ \\
\hline n & 9 & 9 & 10 & \\
$\begin{array}{l}\text { Age } \\
\text { (range) }\end{array}$ & 59 & 63 & 61 & NS \\
Female/male & $(43-80)$ & $(50-88)$ & $(49-88)$ & NS \\
$\begin{array}{l}\text { CK (U/litre) } \\
\text { (range) }\end{array}$ & $11 / 8$ & $2 / 7$ & $6 / 4$ & $\mathrm{P}<0.001$ \\
$\begin{array}{l}\text { CK-MB (U/litre) } \\
\text { (range) }\end{array}$ & $\begin{array}{l}1187-10400) \\
(27-406)\end{array}$ & $\begin{array}{l}(36-229) \\
(1-7)\end{array}$ & & $\mathrm{P}<0.001$ \\
\hline
\end{tabular}

All values are given as median and range except sex. $C K$, serum total creatine kinase; CK-MB, serum creatine kinase MB fraction; MI, myocardial infarction; $n$, number of participants in each group; Non-MI, no evidence of myocardial infarction.

Figure 1 Individual peak plasma $\beta$ thromboglobulin and plasma platelet factor 4 levels in the myocardial infarction group, the group of patients with no infarction, and the healthy controls. Bars show medians. AMI, myocardial infarction group; $B-T G, \beta$ thromboglobulin; NonAMI, patients without myocardial infarction; PF-4, platelet factor 4 .
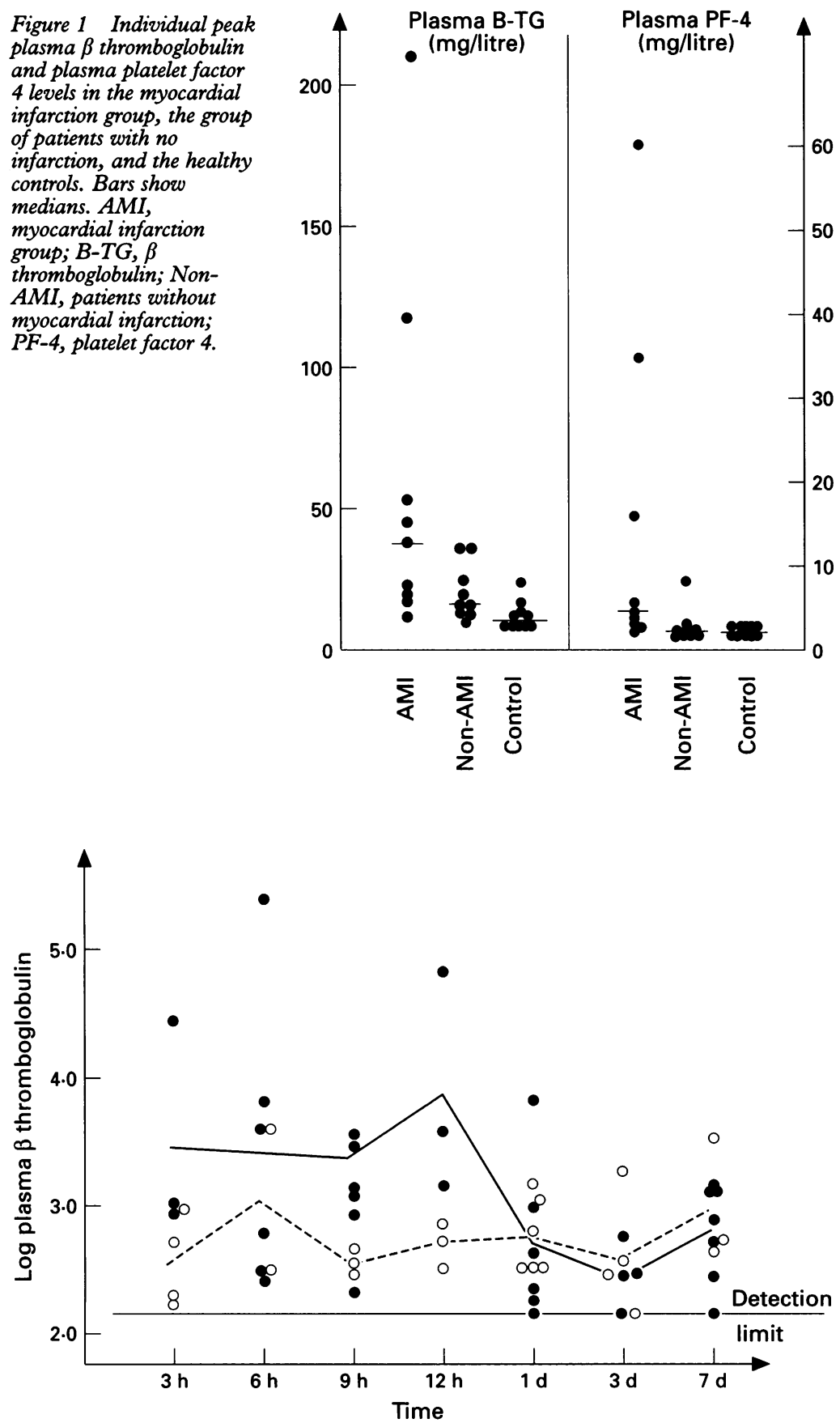

Figure 2 The natural logarithm of plasma $\beta$ thromboglobulin as a function of time elapsed after chest pain. $O$, individual patients in the myocardial infarction group; $\bigcirc$, patients in the group without infarction; (- -$)$ mean of measurements for the myocardial infarction group; (- - - - ) mean of measurements for the group of patients without infarction. glandin $\mathrm{PGE}_{1}$ to stop any in vitro a granule release. The first $5 \mathrm{ml}$ were discarded. Samples were placed on ice for 15-60 min and centrifuged for $60 \mathrm{~min}$ at $4^{\circ} \mathrm{C}$ and $3000 \mathrm{~g}$. The mid-third of the supernatant was collected in cryotubes and stored at $-80^{\circ} \mathrm{C}$ until analysis. Plasma $\beta$ thromboglobulin was measured using a standard radioimmunoassay, sensitivity $9 \mathrm{mg}$ /litre (Amersham Denmark). Plasma platelet factor 4 was measured using a standard enzyme linked immunosorbent assay, sensitivity $2 \mathrm{mg} /$ litre (Hoechst Denmark). All other blood tests were by standard laboratory methods. To reduce in vitro contamination we used a highly standardised procedure, and all measurements were performed by the same staff member. If the plasma platelet factor 4 value amounted to more than one third of the plasma $\beta$ thromboglobulin value, the sample was considered to have been contaminated in vitro and was excluded. ${ }^{10}{ }^{11}$ Only one sample was excluded on this basis.

\section{STATISTICAL ANALYSES}

All continuous data are presented as median and range. The peak value for each patient is defined as the single highest reading of $\beta$ thromboglobulin and platelet factor 4 reached during the $7 \mathrm{~d}$ of investigation. To calculate the significance of difference between the medians of the peak values of the two groups we used the Wilcoxon-Pratt test, and for difference between proportions, the $\chi^{2}$ test. All $\mathbf{P}$ values are two tailed. A $P$ value below 0.05 was considered significant.

\section{Results}

Twenty four patients were invited to join the study. Four declined, leaving 20 patients, 11 with myocardial infarction and nine without. Two myocardial infarction patients did not receive streptokinase and were later excluded. None of the patients without infarction received streptokinase. The myocardial infarction group comprised three anterior, five inferior, and one infero-lateral infarctions. Among the nine patients without myocardial infarction, three had ischaemic heart disease with angina pectoris, but none of the three had ECG evidence of infarction. Six patients had no evidence of ischaemic heart disease on the basis of a negative exercise ECG.

The table shows the age, sex, and creatine kinase values of the two study groups. Leucocytes were significantly increased in the myocardial infarction group compared with the group of patients without infarction $(11.8$ $\times 10^{12} v 7.9 \times 10^{12}$ per litre, $P=0.01$ ) whereas haemoglobin, platelets, serum sodium, serum potassium, and serum creatine were not different in the two groups, $\mathbf{P}>0.05$.

The median peak plasma $\beta$ thromboglobulin in the myocardial infarction group was 37 (12 to 210 ) $\mathrm{mg} /$ litre, substantially higher than in the group of patients without infarction, who peaked at $15(9$ to 36$) \mathrm{mg} /$ litre $(\mathrm{P}<$ 0.01 ), fig 1 . The median peak plasma platelet factor 4 concentration in the myocardial infarction group was $4.6(2.4$ to 60.0$) \mathrm{mg} / \mathrm{litre}$, 


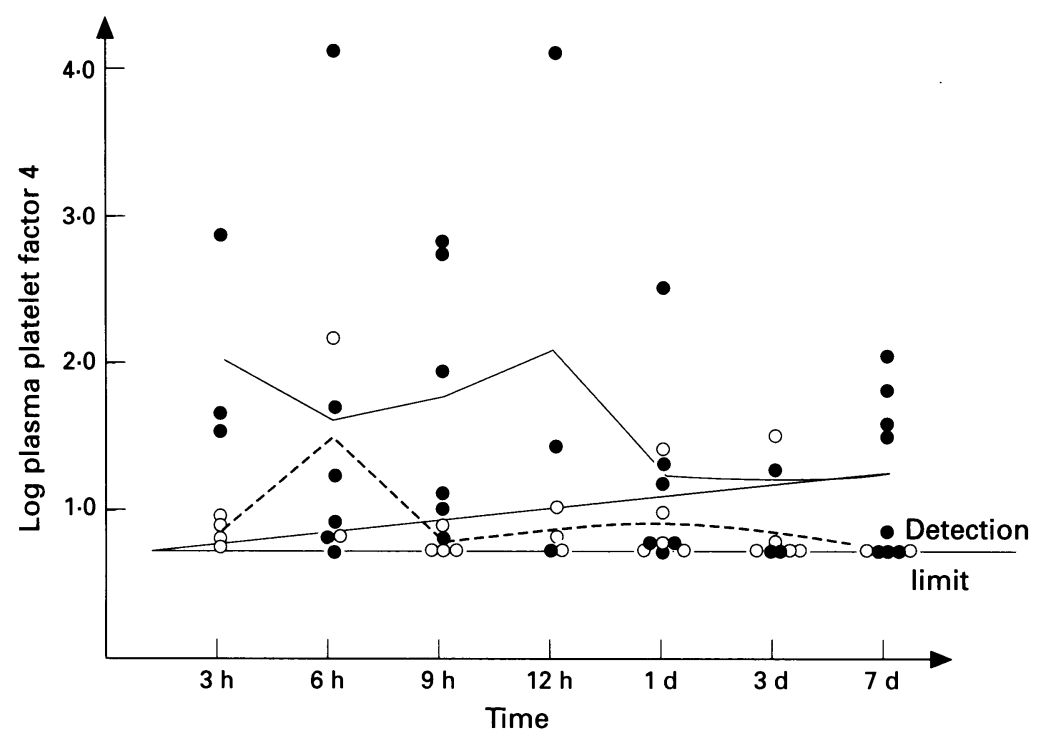

Figure 3 The natural logarithm of plasma platelet factor 4 as a function of time elapsed after chest pain. $\bigcirc$, individual patients in the myocardial infarction group; $\bigcirc$, patients in the group without infarction; $(\stackrel{-}{\longrightarrow}$ mean of measurements for the myocardial infarction group; (-- - - ) mean of measurements for the group of patients without infarction. ment, or a combination of both, that caused platelet release in our infarction group, and it is difficult to conceive of a controlled trial that would separate the two components while being ethically acceptable. Despite many studies in myocardial infarction, the evidence that it can, by itself, induce granule release is suggestive rather than conclusive. ${ }^{3-6}$ The same can be said of streptokinase. ${ }^{1212}$

However, accepting that streptokinase is capable of provoking $a$ granule release, does this necessarily intensify the risk of thrombosis? The question is pertinent to the arterial reocclusion which occurs in a proportion of patients successfully treated with thrombolytic agents. ${ }^{7-9}$ Other things being equal, the answer would be yes, since the classical role of platelet release substances is to enhance the thrombotic process. However, other things are far from equal: at the same time that streptokinase activates platelet release, it also causes platelet aggregability to decrease. In a recent clinical study of acute myocardial infarction, streptokinase caused substantial platelet release while markedly diminishing aggregability. ${ }^{1}$ It is legitimate to question the clinical relevance of any in vitro aggregability measurement. However, Sylvén and coworkers found that plasma fibrinogen was virtually abolished by streptokinase treatment ${ }^{1}$ and suggested that lowered aggregation in thrombolytic treatment is the result of a lack of available fibrinogen, a hypothesis which has immediate relevance to any suspected thrombogenic potential of streptokinase (and other fibrinolytic agents). It may well be that all the actions of streptokinase have, even now, not been revealed, and it would be rash to exculpate the drug in arterial reocclusion simply on the grounds of reduced aggregability; indirect effects on the local circulation could also be involved. However, there seem equally to be no positive reasons for suspecting that thrombolytic agents are likely to increase the risk of thrombosis.

Another important finding in our study is the fact that $a$ granule release occurred while the patients were taking aspirin. This result is in agreement with those of Carrieri et al. ${ }^{13}$ Our result can be explained by the fact that there are many bypassing pathways, causing a release reaction even when the cyclo-oxygenase pathway is blocked. There was, however, no non-aspirin control group, so some damping down of release cannot be excluded.

Attention should be drawn to the wide range of $a$ granule release levels in different individuals (fig 1). As we have been acutely aware from the outset of the technical and handling problems involved in assessing platelet release, and have taken the most stringent precautions to minimise error (see Methods), we are confident that the wide range of responses indicates genuine variation, and not technical error. One can only speculate that the wide spread of results reflects differences in the site or extent of the infarction, or in a variable impact of streptokinase, or that it is simply an inbuilt variation of response to the thrombotic event.
We must, however, be cautious about conclusion, since we had no control group of patients with myocardial infarction who did not receive streptokinase. The observations therefore do not settle whether it is the myocardial infarction, the streptokinase treat- 
This study was supported financially by the Direktor Ib Henriksen Foundation, the Lykfeldt Foundation, the Danish Medical Association Research Fund and The Bønnelykke Foundation.

1 Sylvén C, Karlberg K-E, Chen J, Hagerman I, Egberg N, Bergström K. Enhanced platelet function in acute myocardial infarction is attenuated by streptokinase treatment. F Intern Med 1992;231:595-600.

2 Udvardy M, Harsfalvi J, Boda Z, Rak K. Beta thromboglobulin and increased platelet activation after strep tokinase treatment of acute myocardial infarction. $\mathrm{Am} f$ Cardiol 1992;70:837-8.

3 Ffrench P, McGregor LJ, Berruyer M, et al. Comparative evaluation of plasma thrombospondin, beta-thromboglobulin and platelet factor 4 in acute myocardial infarction. Thromb Res 1985;39:619-24.

4 Galino A, Häberli A, Hess T, Mombelli G Straub PW. Fibrin formation and platelet aggregation in patients with acute myocardial infarction: effects of intravenous and subcutaneou

5 Seitz R, Leising H, Liebermann A, Rohner I, Gerdes H, Egbring R. Possible interaction of platelets and adrenaline in the early phase of acute myocardial infarction. Res Exp Med 1987;187:385-93.

6 Jaffe AS, Lee RG, Perez JE, Geltman EM, Wilner GD,
Sobel BE. Lack of elevation of platelet factor IV in plasma from patients with myocardial infarction. $7 \mathrm{Am}$ Coll Cardiol 1984;4:653-9.

7 Jang I-K, Vanhaecke J, De Geest H, Verstrate M, Collen D, Van Der Werf F. Coronary thrombolysis with recombinant tissue-type plasminogen activator: patency rate and regional wall motion after 3 months. $7 \mathrm{Am}$ Coll Cardiol 1986;8:1455-60.

8 Ganz W, Geft I, Shah PK. Intravenous streptokinase in evolving acute myocardial infarction. Am 7 Cardiol 1984; 53:1209.

9 Harrison DG, Ferguson DW, Collins SM, et al. Rethrombosis after reperfusion with streptokinase: imporance of geometry of residual lesions. Circulation 1984; 69:991-9.

10 Kaplan KL Owen J. Plasma levels of beta-thromboglobulin and platelet factor 4 as indices of platelet activation in vivo. Blood 1981:57:199-202.

11 Kaplan KL, Owen J. Plasma levels of platelet secretory proteins. Crit Rev Oncol Hematol 1984;5:235-55.

12 Bertolino G, Noris P, Previtali M, et al. Platelet function after in vivo and in vitro treatment with thrombolytic

13 Carrieri P, Orefice G, Indaco A. No effect of acetylsalicylic acid on $\beta$-thromboglobulin and platelet factor 4 plasma levels in patients with transient ischaemic attacks. Stroke 986;17:1153-5. 